COMMENT. Seizures in the neonate with hypoxic-ischemic encephalopathy are often subtle, characterized by swimming, rowing movements, apneic spells, opisthotonus, or clonic movements. (Volpe JJ. Observing the infant in the early hours after asphyxia. In Gluck L, ed. Intrauterine asphyxia and the developing fetal brain. Chicago, Year Book Medical Publ, 1977).

In newborn rats, seizures induced by electroshock vary with strength of current and with age. In 10-day-old rats, the animal exhibits hyperkinetic behavior, breaststroke swimming movements, and transient opisthotonus. At 20 days, tremor of head, and catatonic posture; and at 30 days, the seizure is a generalized symmetrical clonus with loss of posture. A stronger current is required to induce a maximal tonic clonic seizure in rats older than 15-20 days. In rats aged 1 to 15 days, a maximal tonic seizure cannot be elicited. The increased severity of seizure response with increase in age is correlated with development of brain carbonic anhydrase. (Millichap JG. Development of seizure patterns in newborn animals. Significance of brain carbonic anhydrase. Proc Soc Exptl Biol Med 1957;96:125). If slightly older rat pups ( $>6$ days) had been employed in the Helsinki experiment, a more definitive seizure endpoint might have been elicited. The following clinical report supports the association of PCO2 and outcome of HIE in neonates, but raises questions regarding a seizure as the measure of brain damage and disability.

Pappas A and colleagues (J Pediatr May 2011;158:752-758) in the Eunice Kennedy Shriver NIH Neonatal Research Network report that infants exposed to low $\mathrm{PCO} 2$ concentrations within the first 16 hours of life during whole body cooling were at increase risk of death/disability. Disability was directly related to the degree and severity of hypocarbia. Both minimum PCO2 and cumulative PCO2 $<35 \mathrm{mmHg}$ were associated with poor outcome at 18-22 months of age. The occurrence of clinical seizures was not significantly associated with death or disability. The infants had received ventilatory resuscitation or assistance at birth, and this may have contributed to hypocarbia. Further investigation will determine whether hypocarbia is a modifiable risk factor or simply a marker of poor outcome. Clyman RI, in an editorial (Jrnl Pediatr 2011;158:A2), asks "Should cooled infants be started on lower initial ventilator settings or weaned more rapidly?" "Will avoidance of hypocarbia improve outcome?"

\title{
ANTICONVULSANT DRUGS
}

\section{FOLIC ACID SUPPLEMENT PREVENTS PHENYTOIN-INDUCED GINGIVAL HYPERPLASIA}

Researchers at the Division of Pediatric Neurology, Neurosciences Centre, New Delhi, India, performed a randomized, double-blind, placebo-controlled 6-month trial of oral folic acid supplement $(0.5 \mathrm{mg}$ /day) in children aged $6-15$ years treated with phenytoin for epilepsy. Of 62 patients who received folic acid supplement, $21 \%$ developed gingival hyperplasia whereas of 58 receiving placebo, $88 \%$ developed hyperplasia $(\mathrm{p}<0.001)$. Risk reduction of phenytoin-induced gingival hyperplasia by folic acid was $67 \%$. The mean phenytoin dose was similar $(6.8$ and $7.0 \mathrm{mg} / \mathrm{kg} / \mathrm{day}$ respectively) in folic acid and placebo groups at the end of the study. Mean serum levels of phenytoin were therapeutic $(13 \mathrm{mcg} / \mathrm{ml})$ in both groups. Seizures were symptomatic, 
and $76 \%$ were due to neurocysticercosis. (Arya R, Gulati S, Kabra M, Sahu JK, Kalra V. Folic acid supplementation prevents phenytoin-induced gingival overgrowth in children. Neurology April 12, 2011;76:1338-1343). (Response and reprints: Dr Sheffali Gulati, Department of Pediatrics, AIIMS, Ansari Nagar, New Delhi, 110 029, India. E-mail: sheffaligulati@gmail.com).

COMMENT. Gingival hyperplasia associated with phenytoin treatment of epilepsy is reported in as few as $3 \%$ of cases (Lennox WG, 1940) to as many as $78 \%$ (Gardner AF et al, 1962). It occurs more frequently in children than in adults. Numerous mechanisms have been proposed but few of proven significance. Other hydantoin anticonvulsants (mephenytoin, ethotoin, and albutoin) cause little or no gingival hyperplasia. The above investigators have discovered an important and correctable factor in the mechanism in their clinic population. The authors allude to a lack of dental hygiene in a high proportion of patients, a known contributing factor associated with tissue inflammation and irritation. Hyperplasia does not occur in edentulous adults. Phenytoin has an affinity for gingival tissue, and its effect on collagen and keratin in connective tissue has been used in the promotion of healing of wounds and leg ulcers (Shafer WG et al, 1958; Houck JC et al, 1972). In addition to man, only the ferret is susceptible to phenytoin gum hyperplasia, an interesting companion in science. Mechanisms largely disproven include a deficiency of ascorbic acid, adrenocortical dysfunction, and allergy (Gardner, 1962). Hyperglycemia induced by phenytoin, an effect discovered in our neurology research laboratories at Children's Memorial Hospital (Belton NR et al. Epilepsia 1965;6:243-249), warrants investigation as a potential contributing cause of gingival inflammation and overgrowth.

\section{VALPROIC ACID-ASSOCIATED LIVER FAILURE}

Researchers at the University of Maryland School of Medicine, Baltimore, and other centers analyzed the records of 17 children undergoing liver transplantation (LT) for valproic acid-associated liver failure (VPA-ALF) and 98 with ALF caused by other drugs (non-VPA-drug-induced acute liver failure [DIALF]. A case report of an 18month-old boy with ALF during treatment with VPA is diagnosed with a mitochondrial disease with 2 distinct heterogeneous missense mutations in DNA POLGI. He was declined as a candidate for LT because of the poor prognosis of LT after VPA-ALF, and he recovered after discontinuation of VPA and initiation of carnitine therapy. In the record review of 17 patients transplanted for VPA-ALF, 14 (82\%) died within 1 year of LT, whereas the 1-year survival for children who underwent LT for non-VPA-DIALF was $69 \%$. $(\mathrm{P}<.0001)$. Except for a lower median alanine aminotransferase level at transplant in VPA-ALF compared with non-VPA-DIALF (45 vs $1179 \mathrm{IU} / \mathrm{L}, \mathrm{P}=.004$ ), pre- and post-transplant parameters of the two groups were comparable. Median post-LT survival time for VPA-ALF was 2.8 months. Children undergoing LT for VPA-ALF have a significantly lower survival probability than children with non-VPA-DIALF. VPA-ALF may represent an unmasking of mitochondrial disease and should be contraindicated for LT, even in absence of mitochondrial disease. In this series, an association with mitochondrial disease was not confirmed. (Mindikoglu AL, King D, Magder LS, Ozolek JA, Mazariegos GV, Shnieder BL. Valproic acid-associated acute liver failure in 